\title{
USING DIFFUSION OF INNOVATION THEORY TO IMPLEMENT ELECTRONIC HEALTH RECORDS FRAMEWORK IN AFGHANISTAN
}

\author{
Abdul Wahid Samadzai ${ }^{1}{ }^{\square}$, David Lamas ${ }^{2}$ 四 \\ ${ }^{1} \mathrm{PhD}$, Student at School of Digital Technologies at Tallinn University, Europe \\ ${ }^{2}$ School of Digital Technologies at Tallinn University, Europe
}

DOI: https://doi.org/10.29121/ijetmr.v8.i2.2021.857

Article Citation: Abdul Wahid

Samadzai, and David Lamas. (2021).

USING DIFFUSION OF INNOVATION

THEORY TO IMPLEMENT

ELECTRONIC HEALTH RECORDS

FRAMEWORK IN AFGHANISTAN.

International Journal of Engineering

Technologies and Management

Research, 8(2), 70-78.

https://doi.org/10.29121/ijetmr.v8 .i2.2021.857

Published Date: 28 February 2021

Keywords:

Innovation

Record

Framework

Afghanistan

Hospital

Clinic

\begin{abstract}
In an environment of widespread demand in the healthcare system for the provision of equitable, achievable and safe healthcare, the use of information communication technology (ICT) is individual of the identified solutions to meet such expectations. The Electronic Health Record (EHR) is a main instrument towards achieving well healthcare using such technology, although, across the world EHR implementation has experienced a great failure rate. The aim of this research is to move from paper-based health record to some electronic health record. For achieving this aim, the researcher used diffusion of innovation theory.
\end{abstract}

\section{INTRODUCTION}

Afghanistan is a developing country consisting of wide rural and urban areas. The Afghan healthcare system has public and private health sectors. People in Afghanistan are increasingly using computers in various aspects of life, although mainly for general purposes, such as saving information locally in offices, printing, or socialising. In terms of medical care, only a limited set of digital technologies used for diagnosis, treatment and disease control.

A major challenge in developing countries seen in the economic area[1]. This leads to poor access to quality health services. In reality, the welfare of the country's health system nearly relevant to its economic status. For this reason, improving economic conditions will lead to the expansion of the health system. On the other hand, progress in health leads to economic growth. Better economic conditions are essential for any developing country, and therefore improvements in the health system, especially those achieved via e-health solutions, are especially relevant for a good economy[2].

According to Anwar (2011), the significant problem in organizing education is the establishment and performance of safety information technology in underdeveloped countries dependent to time limitations and financial. Converting any system is a hard function and not be done in a short time. Barriers such as a shortage of skilled worker, infrastructure and prices, along with other affects, such as an initial reduction in productivity due to

(C) 2021 The Author(s). This is an open access article distributed under the terms of the Creative Commons Attribution License, which permits unrestricted use, distribution, and reproduction in any medium, provided the original author and source are credited. 
adaptation to new technology surroundings, impose powerful boundaries on the introduction and adoption of new health technologies. It takes years to perfect the conversion process.[3].

According to James G. Kahn, Joshua S. Yang (2010), the big challenge in developing countries related to education, most people live in villages where levels of education are low. The result is that people often cannot read or write. They do not possess sufficient knowledge about information technology and cannot use a computer or other electronic devices as a result [4].

Patient knowledge is necessary for the functioning of e-health systems. Transitions from existing paper-based approaches to computerised information systems is not possible without providing knowledge to health experts.

The aim of this research is implementation suggested framework for EHR in the Ministry of Public Health of Afghanistan, based on the understanding from the literature and the skills of the other developing countries

1) How to transfer from paper-based system to electronic health record management in Afghanistan?

- How could use diffusion of innovation theory for implantation EHRs framework?

- What were the big problems of moving from paper-based to EHRs?

\section{LITERATURE REVIEW}

The Ministry of Public Health in developing countries is the central governmental body, whose role is to identify health needs and priorities, formulate national policies and strategies, monitor performance, manage and mobilise resources for the public health sector and establish and enforce rules and regulations for the private sector [5].

A number of developing countries have similar challenges in their health systems as the ones in Afghanistan that described earlier in chapter one. For example, being able effectively use computerised information systems require having the necessary skill set and experience. Thus, training becomes important for successful use of new technology and provides the possibility for overcoming deficiency of skilled workforce. One of the required training packages should focus on construction of reliable databases. The lack of training in this area could lead to barriers in decision-making processes and evidence-based practices. Provision of quality training, however, requires significant investment in terms of time and money [6].

According to Kalogriopoulos et al. (2009), developing countries do not have a well-developed health system infrastructure. On the other hand, there are high rates of terminal illnesses, such as HIV and malaria, which leaves millions of people in a major need of medical assistance. It estimated that $95 \%$ of the population of developing countries could potentially affected by these illnesses. Devastating illness like HIV need constant care and treatment to held on a manageable level. For this reason, basic paper-based medical record management practices are not sufficient and should replace by more effective electronic medical records (EMRs) systems [7].

Keeping medical records in paper-based form has been sufficient in the past, but today in the 21st century there is a need for new and better systems. Over time, paper-based systems have become increasingly inefficient and are continuously failing to meet the evolving needs of the health care providers [8]. With a paper-based system, communication between different parties involved in the provision of health is extremely difficult, especially in developing countries. In United States for example, a paper-filed medical record may scan and sent to another care provider or sometimes faxed to wherever it needs to go. In developing countries, if a paper record medical record needs to see by a different care provider or someone at a different location, then that paper document would need to deliver to this new location by hand [9]. This method takes a lot of time and is inefficient, adding a lot of overhead along with the time it takes to conduct medical tests. For example, if a patient needs to get some kind of test, he or she needs to carry the paper record to the lab where the test would be administered, and then, these test results in paper form would have to make their way all the way back to the doctor in order to be analysed. It is obvious that paper-based medical record keeping does not meet the needs of an efficient health care model[10].

Despite the documented advantage of the EMR system, most medical encounters with patients still recorded by hand by using a paper record, and there is a reason for that. Traditional paper records still considered familiar to users, they are portable, recording and browsing information for patients with not complex conditions is easy[11]. There is also a sense of ownership of paper records, due to their being only one copy, which increases the sense of security. However, it should note that this might well be a false sense of security [12]. 


\section{SUGGESTED FRAMEWORK FOR EHRS IMPLEMENTATION}

The aim of this research is to use EHRs in instead of paper-based system in the Ministry of Public Health (MoPH) in Afghanistan. The main responsibility of the MoPH in Afghanistan is to implementation EHRs framework in all provincial's hospitals and clinics[13]. Researcher suggest framework for EHRs implementation in hospitals and clinics. Figure one, illustrate framework for EHRs implementation.

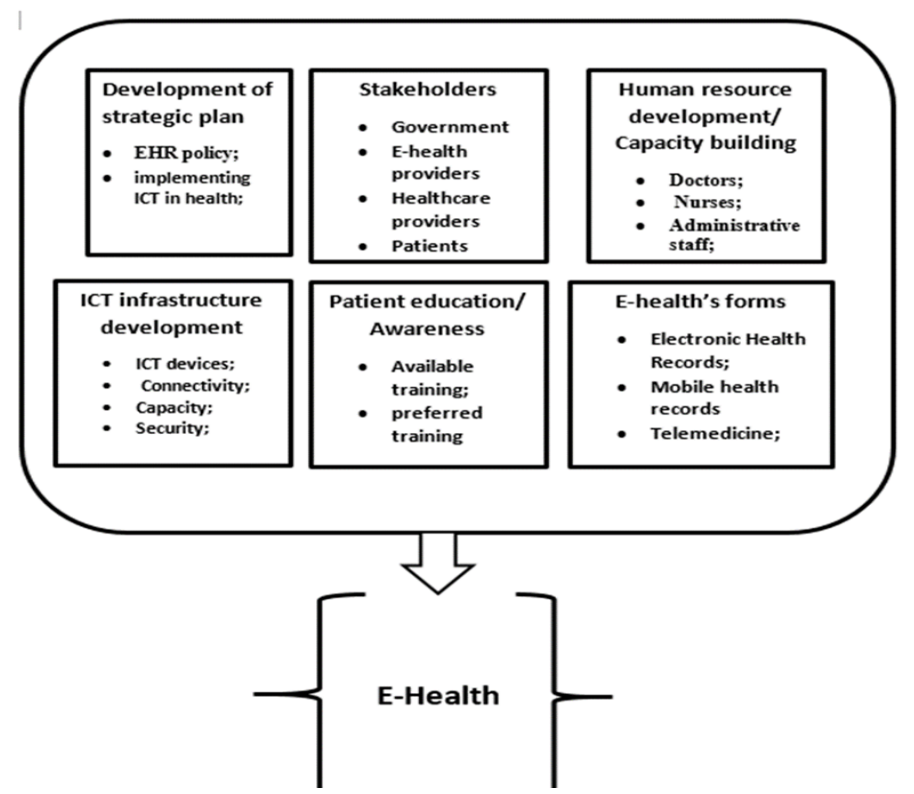

Figure 1: A framework for EHRs implementation

Figure one, illustrate proposed framework for EHRs implementation in Afghanistan. This framework has six main parts, the first part is development of strategic plan (EHR policy, implementing ICT in health). In second part focus on stockholders of proposed framework, government, E-Health providers, healthcare providers and patients are stockholders for EHRs framework. The third part of EHRs implantation framework is human resource development/ capacity building, the third part focus on capacity building of doctors, nurses and administrative staff. The implementation of ICT in health needs to have professional staffs in hospitals and clinics in rural and urban area. The fourth part of proposed framework discussed about ICT infrastructure development. This part is including in ICT device, connectivity, capacity and security. Fifth part discussed about patient education/ awareness, patient need to some short and long-term ICT training course for increasing their knowledge. The sixth part focus on EHRs forms, mobile heath records telemedicine.

\section{METHODOLOGY}

The main research methodology used in this research is qualitative research. The initial step in this study is to conduct focus group interviews with physicians, nurses, managers, and patients. Interviews conducted at several hospitals in Afghanistan to illustrate the problems relevant to EHRs development and healthcare in the country in general.

In this research, the interviews complemented by a focus group interviews study that goal to provide an indepth description of daily life and performance at three health centers - two private hospitals and a public hospital.

The researcher conducted to stockholders, the researcher wrote stockholder need and how they interested to this research in the first part of section. The researcher created a framework for EHR implementation in Afghanistan' health system. Researcher had interview with stockholders for validation of the framework.

In this research, the researcher did focus group interview with 41 experts in six groups. Five groups included seven interviewers and one group was include six experts. In this round of interviews conducted in Ministry of public health and some public hospitals in Kabul city. Interview conducted with policy makers in Ministry of public health, doctors, nurses and hospital staffs. In this round, twelve interviewees were policy makers in Ministry of 
Abdul Wahid Samadzai, and David Lamas

public health 8 of them were male, four of them were female and policy makers had different working experiences. Fourteen were doctors in hospitals, ten of them were male, four were female, and doctors had different work experiences. Eight experts were nurses, five nurses were female and three of them were male. Seven-interviewed expert were hospitals management staffs, all of them were male.

Researcher used stakeholders' (interviewed experts) opinions to validate the framework for EHRs implementation. The interviewed experts (stakeholders) were including, Administrators, doctors, nurses and IT staffs. The interviewed experts filled out the questionnaire. Interviewed experts were required to answer A as (Excellent), B - (Good), C- (Adequate) and Not acceptable to the questions.

\section{DIFFUSION OF INNOVATION FRAMEWORK EHR}

According to James W. Dearing and Jeffrey G. Cox (2018), the diffusion of innovation theory has broadly applied to the study of information technology (IT) innovations for more than the last decade and has provided insight into the adoption, implementation, infusion, and diffusion of IT innovations. Diffusion of innovation has five steps: knowledge stage, persuasion stage, decision stage, implementation stage, conformation stage [14].

The knowledge is the main step of diffusion of innovation theory for implementation the new idea in information technology. The knowledge step happens when the researcher or a group of researchers have a new opinion about a possible innovation and the "know-how" for the construction of its [15]. For the EHR implementation framework in Afghanistan, it is very important to have an idea about personality characteristics of IT staff in hospitals, social characteristics like as (using ICT), and communication integration. In the knowledge stage, we answer what, how and why questions.

In the following we answered to "what?":

The main aim of this research is the innovation of implementation of the EHRs framework in the Ministry of public health in Afghanistan.

The diffusion of innovation of proposed framework give answer to this question "How to transfer from paperbased system to electronic health record management in Afghanistan?". The framework has six parts development strategic plan, stockholder, human resource development/ capacity building, ICT infrastructures development, patient education/ awareness and E-health's forms.

The second part of the knowledge stage is how-to-knowledge; the stockholders know how diffusion of innovation ICT can be in the health system. The diffusion of innovation, having strategic plans in the health system, gives the ability to policy makers in the Ministry of public health to adopt EHRs implementation framework equal in all 34 provinces in Afghanistan.

Innovation of ICT infrastructures development is the other part of framework EHRs implementation. The innovation of ICT infrastructures is including ICT devices like hardware and software, the diffusion of innovation connectivity hospitals and clinics to each other. The innovation of security systems in all public and private hospitals and clinics.

Human resource development/ capacity building is the main part of diffusion of innovation framework EHRs implementation in Afghanistan, without having professional IT staff in all provincial hospitals and clinics it is not possible to adopt the framework of EHRs in the Ministry of public health.

The diffusion of innovation in some short and long-time courses for IT staff, doctors, and nurses to increase their knowledge for using new ICT infrastructures in the health system. Training courses are necessary to updating knowledge of doctors, nurses, IT staff and patients for using new technology. Doctors, nurses and IT staff in some provincial hospitals and clinics have not enough knowledge about using ICT infrastructures like computers, fast internet, routers, and switches.

Social characteristics are very important for the implementation of the EHR in the Ministry of Public Health in Afghanistan. Social characteristics are including in a cultural status, economic, quality, accountability, accessibility, cost and rather. Each province's culture is especial, and attention to personality traits is essential to achieving innovation in EHR implementation in Afghanistan.

The answer to why question is as:

In current time, the cost of health services is very high. The diffusion of innovation framework of EHRs implementation is necessary to decrease the health services cost. Time is the main factor for patients and health 
Using Diffusion of Innovation Theory to Implement Electronic Health Records Framework in Afghanistan

managers, then the diffusion of innovation framework for EHRs implementation, could help to decrease lack of time. The adopted EHRs framework could help to move from paper-based to electronic health systems.

\subsection{PERSUASION}

\subsubsection{RELATIVE ADVANTAGE}

The 99 percent stockholders (interviewers) mentioned to the relative advantage of the diffusion of innovation framework for EHR implementation in Afghanistan. In this way, the Ministry of Public Health could provide good services to patients and hospitals staffs and clinics staffs in all regions of the country. Through the EHR, patient health records and records can be easily access, as well as the problem of loss of time. The relative advantages of using ICT infrastructure to implement EHR in Afghanistan are as follows:

- The medical staffs like as (Doctors, nurses, IT staffs and patients) could have easily access to data at their convenience;

- The steps have decreased and the health providers could provide the best health service to individuals. With having good internet connections and the essential internet instrument like as (switches, routers and servers), all very significant to provide better services to patients;

- $\quad$ Reduced lack of time;

- Having relationship and share some data between all hospitals and clinics;

The relative advantage identified by $100 \%$ interviewer (stockholders). There is a clear need for patients to participate in training courses on the use of new technology in the health care system. The reason is that in some provinces, citizens do not take enough education. To solve this problem, the government must create short-term and long-term courses to increase the level of education of the people.

\subsubsection{COMPATIBILITY}

Compatibility to diffusion of innovation EHR implementation framework discussed in focus group interviews. The interview experts admitted the innovation in the healthcare organisation by using and implementing the EHRs instead of the paper-based healthcare system. Several policy makers like as doctors and IT staffs experienced the necessity for implementing EHRs and understood the interests of using ICT in the healthcare system for instance, the capability to provide information to patients in a protected technique and in local languages like Dari and Pashto.

The experience of interviewee displays that it is essential to innovate in the healthcare organisation, and instead of the paper-based system, the electronic health system must be use. The $98 \%$ interviewed expert displays, which the attitude of the medical staffs is very important for the efficient admission of the ICT infrastructure in the healthcare system. It is why this is essential to comfort and facilitate adaptation to cultural values, beliefs and attitudes. Compatibility of information and communication technology must be in line with personal styles and skills, and ultimately significant satisfaction and continuous use must be achieved.

The concept of compatibility was identified in completely the situations of focus group interviews. Established on the interview a strong and clear essential recognised for patients to participate in training courses related to the usage of innovative and new technology in the healthcare system local language (Dari and Pashto). This is because in more developing countries persons do not take enough education. To talk this, the administration essentials to improve short and long-time courses to raise individuals' education levels and especially for women.

\subsubsection{COMPLEXITY}

A framework for EHRs implementation innovation might confront hospitals and clinic members with the challenge of changing their patient registration methodology to integrate the technological innovation into their instruction; therefore, it might have different levels of complexity. If hardware and software are user-friendly, then IT managers adopted successfully for the delivery of medical materials. The user interface might be friendly and designed in local language as Dari and Pashto. 
The electronic health record complexities are a useful cause affecting appropriation. Incoming the information of each personal patient into electronic health record is exact and complex, that means which application designed to it end, must reject complexity.

The maximum score was of complexity, said in $80 \%$. Partial interview experts are facing complexities in using information communication technology's infrastructures approximately in the absence of high-speed networks in rural and urban regions, the non-existence of expert IT workforce, and the non-existence the information communication technology's infrastructures in overall.

The complexity was recognize in $78 \%$ interviewers. Complexity discussions to the problem of admitting or learning to usage a fresh awareness and knowledge. It might affect the speed at which innovations that essential to improvement fresh skills and understandings arrive appropriated. The execution of EHRs wants considerable learning since they are multi-aim and complex systems. The hospitals workforce and patients essential training courses for using the information communication technology in the healthcare system.

\subsubsection{TRIALABILITY}

Trialability of diffusion of innovation framework for EHRs implementation is major part of this research. The policy-makers (decision makers) in Ministry of Public Health of Afghanistan and hospitals (clinics) which have until now to adopt the electronic health record will study from the knowledges of hospitals in developed and developing nations which have earlier implemented electronic heath records and might be additional expected to take up the fresh technology.

Ninety two percent of interview experts discussed about the trialability of EHRs implementation framework in Afghanistan. Trialability done by several interview experts (stockholders) from examine out different ICT infrastructures for comfort the move from the paper-based to EHRs management system, for instance by using webbased electronic health record systems in hospitals and clinics. Others usage mobile-based systems for electronic health record. For instance, of using Trialability to support adoption. ICT advantage from the Trialability of their product in two ways, first as a new ICT infrastructure introduced, free samples make IT staff aware of the product. The second once the IT infrastructures is well accepted, free sample help IT staff introduce doctors, nurses and patients to a new ICT infrastructure.

By expert suggestion conferences and trade displays for health and information technology specialists, technology sellers give demonstrations that walk persons over the procedure of the innovation and hands-on trial using it on imitation of the model. In the First-hand medical material organizations could tried out with little promise over websites and application services that do not need fully installing or converting to the new method.

\subsubsection{OBSERVABILITY}

Observability mentioned in $95 \%$ of three interview. Observability attained by several interview experts (interviewee) via examination out many ICT infrastructures for accepting the move from the paper-based to EHRs management system, for example by usage web-based EHR systems in hospitals and clinics. Others usage mobilebased systems for electronic health record. In the observability step, some diffusion of innovations is not easy to observe and thus might diffuse further slowly. This is tough to observe a person's experience with IT and even tougher when the observer does not identify what to aspect for.

A small number of innovations improve the long-term framework EHRs implementation results but display no instant change in outcomes. Instances are devices to lower the danger of main or recurrent actions. Unknown new technology produces observable outcomes that need more expertise to understand them and create them comprehensible, that technology may be slower to diffuse. Instances are novel devices that are expect to benefit a small, but main, sub people within various categories.

\subsection{DECISION}

All interviewed experts (stakeholders) demonstrate, the developed EHR framework excellently provides solutions to the main problems (challenges) and also all interviewed experts are willing to implement the 
recommendations in the proposed EHRs framework and would recommend the framework to other organizations (private health sectors).

All stakeholders (interviewed experts) agree that EHR implementation is important and beneficial in the health sector in Afghanistan. All the interviewed experts (stakeholders) said, an important diffusion of innovation in the health sector is the implementation of EHRs. The Ministry of public health in Afghanistan needs to have the best strategy plan for EHR innovation in hospitals, because without having a good strategy plan the implementation of EHR is not possible in all 34 provinces and villages.

The 99 percent of interviewed experts agreed to have professional human resources for the implementation of EHRs in public health sectors and private health sectors. The lick of human resources in hospitals and clinics is a big problem. In some provinces, hospitals and clinics do not have enough professional staffs (doctors, nurses and IT staff).

Interviewed experts said, people and patients need long and short time training courses in using ICT and some programs in television and in radio for increasing knowledge. The Ministry of Education must add lessons in school's books.

Stakeholders said the implementation of EHRs in Afghanistan need to have fast internet connection in all provinces and villages. Internet connection is a very important instrument for EHRs implementation so the Ministry of health needs to have a strategy plan to increase and send internet equipment to all villages.

Stakeholders illustrated, people need to have fast and low-cost internet connection to share information with his/ her doctor. The internet cost is very high and connection is very slow. High-speed internet connection can help with running online training courses for hospitals and clinic staff in villages.

Stakeholder said, improving patient knowledge is necessary for a good e-health system. To moving from a paper-based health record system to an electronic information, system is not feasible without providing learning to health experts (professionals). There are no skilled medical employees in the broad area of specializations and training programs to increase basic health services.

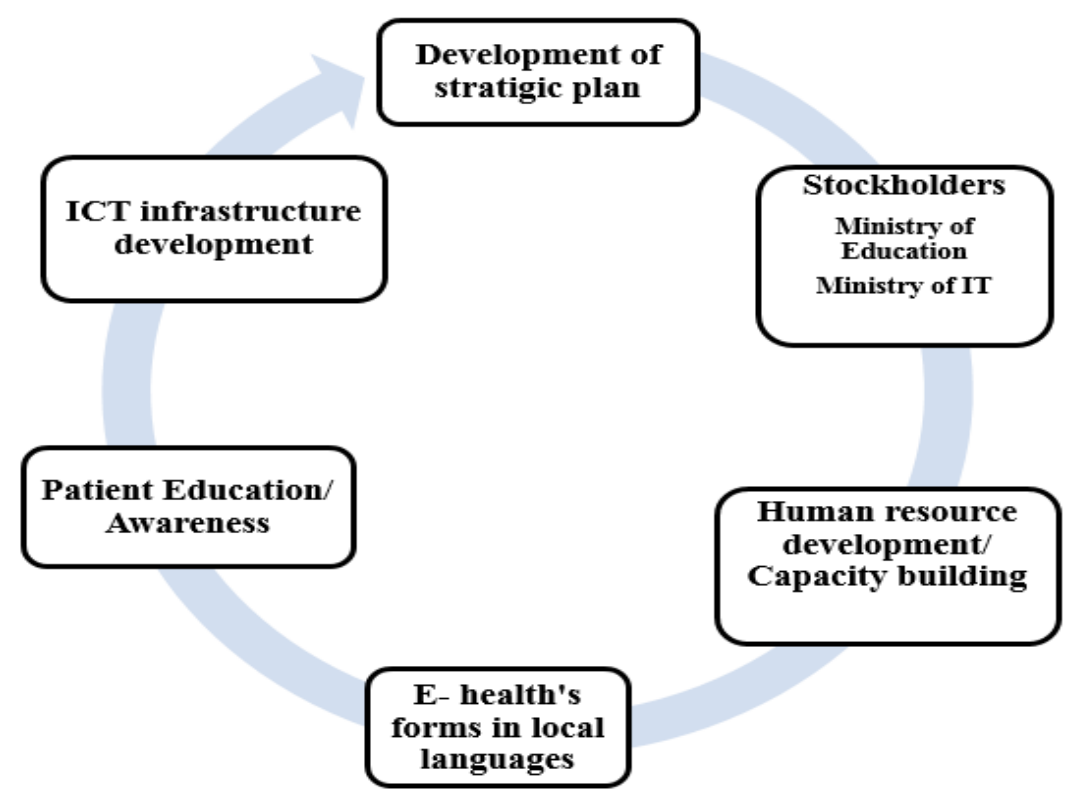

Figure 2: Framework after validation

Figure 2 illustrated, $100 \%$ interviewed experts said people and patients need friendly and easy applications for EHRs in local languages such as (Dari and Pashto). Some people have not enough knowledge to write and read in English language then patients and users do not use applications for EHRs in English better.

Figure 2 illustrated, $98 \%$ interviewed experts mentioned adding the Ministry of Information Technology and Ministry of Education to the stockholder part of framework EHRs implantation in Afghanistan. Interviewed experts said, help from the Ministry of IT and the Ministry of Education are necessary to adopt EHRs implementation framework. 
Abdul Wahid Samadzai, and David Lamas

\subsection{IMPLEMENTATION}

Implementation is in the fourth position of diffusion of innovation operation. At implementation step, working on innovations can vary depending on the situation. In this view, the usefulness of the innovation is determined and additional information is review. Implementation occurs when individuals or other decision-making teams combine the new idea and start using the EHR.

\subsection{CONFIRMATION}

The verification (confirmation) step occurs when population of people search to finalize decisions they have already built. However, if persons are expos to conflicting messages about innovation, a decision may exchange. Every of these steps discussed because it relates to the implementation of the EHR to support the existing health record management (which is currently enable by paper-based systems).

\section{CONCLUSION}

Afghanistan government has responsibility to do same health services to all citizens. Government promised to move health system from paper-based system to electronic system, Afghanistan government need to have health electronic system like as framework for implementation EHR in all provinces. Afghanistan government was interested to have easily, fast and cheaper health services to all people.

The Ministry of public health is health provider for all Afghan people, stakeholders are policy maker work at the Ministry of public health (some doctors of hospital are policy maker). Policy maker tooled, the Ministry of public health in Afghanistan need to have strategic plan and policy of adoption of EHR in all provinces hospitals. Policy makers involved to make strategic plan for to give good and easily health services to all citizen in Afghanistan.

The stockholders (doctors and nurses), stockholders mentioned to the ministry of public health need to move from paper-based record system to electronic health record for gave good, easily and low-cost health services to people. Also, for implementation of EHRs need to infrastructures, professional staff, some training courses for doctor, nurses and hospital staffs. Stockholders (doctor and nurses), were involved in our research, I asked some questions about my research from the stockholders (doctors, nurses and hospitals staffs) and I got information and idea to use in my research.

The hospital's IT staff and managers have responsibility to provide electronic services into all hospital. IT managers and IT staffs are stockholders for implementation EHRs in Afghanistan health system. The hospitals work staff and managers were involved in our research. I had some questions about registration system in hospitals, IT infrastructures and using computerized system for patient's registration, training course for increasing their knowledge then managers and staffs prepared and give information to me. Managers and staff mentioned to points that they need like as internet connection, ICT infrastructures, and some short and long-time training courses. Managers and staff were interested to have a good framework for implantation of EHR in hospital.

Patients were interested to have fast and cheaper health system services. Patients could see their histories, so patient need to know about using the EHRs, for this purpose patient need to some training course. Patient mention to village's clinics needs to internet connection and ICT infrastructures in hospital and clinics in all villages.

All interview experts (Ministry of Health policy makers, physicians, nurses, management staff, patients) said which the Afghan health system need to has a useful hospital information system in all hospitals and clinics to establish the best services to the citizens of Afghanistan. In a focus group interview with policymakers at the Ministry of Health, they said that a national policy is very important for the health service. Special questions from policy makers asked about creating the useful EHR policy for hospitals and clinics in all provinces. Policymakers noted that it is necessary to implement national policies. To formulate such a policy, policymakers must focus on the challenges of each province and village. All stockholders said that they help to implantation of EHR n hospitals in all provinces.

\section{SOURCES OF FUNDING}

This research received no specific grant from any funding agency in the public, commercial, or not-for-profit sectors.

International Journal of Engineering Technologies and Management Research 
Using Diffusion of Innovation Theory to Implement Electronic Health Records Framework in Afghanistan

\section{CONFLICT OF INTEREST}

The author have declared that no competing interests exist.

\section{ACKNOWLEDGMENT}

None.

\section{REFERENCES}

[1] J. Scholl, S. Syed-abdul, and L. Awad, "A case study of an EMR system at a large hospital in India: Challenges and strategies for successful adoption," J. Biomed. Inform., vol. 44, no. 6, pp. 958-967, 2011, doi: 10.1016/j.jbi.2011.07.008.

[2] K. R. Shah, "Establishment of Electronic Health Records in Developing Countries," vol. 136, no. 11, pp. 20-24, 2016.

[3] F. Anwar, "Barriers in Adoption of Health Information Technology in Developing Societies," vol. 2, no. 8, pp. 40-45, 2011.

[4] and J. S. K. James G. Kahn, Joshua S. Yang, “'Mobile' Health Needs and Opportunities in Developing Countries,” 2010.

[5] B. A. Y. Al-nassar, M. S. Abdullah, W. Rozaini, and S. Osman, "Overcoming challenges to use Electronic Medical Records System (EMRs) in Jordan Hospitals,” vol. 11, no. 8, pp. 51-58, 2011.

[6] S. T. Mm. Shrikant I. Bangdiwala, Sharon Fonn MBBCh, "Workforce Resources for Health in Developing Countries," 2006.

[7] N. A. Kalogriopoulos, J. Baran, A. J. Nimunkar, and J. G. Webster, "Electronic Medical Record Systems for Developing Countries: Review," 2009.

[8] S. Ajami, S. Ketabi, S. S. Isfahani, and A. Heidari, "Readiness Assessment of Electronic Health Records Implementation," vol. 19, no. November, pp. 224-227, 2011, doi: 10.5455/aim.2011.19.224-227.

[9] S. S. Ravindra, R. Chandra, and V. S. Dhenesh, "A Study of the Management of Electronic Medical Records in Fijian Hospitals," 2015.

[10] H. S. F. Fraser, P. Biondich, D. Moodley, S. Choi, B. W. Mamlin, and P. Szolovits, "Implementing electronic medical record systems in developing countries," Inform. Prim. Care, vol. 13, pp. 83-95, 2005, doi: 15992493.

[11] N. Huba and Y. Zhang, "Designing patient-centered personal health records (PHRs): Health care professionals' perspective on patient-generated data," J. Med. Syst., vol. 36, no. 6, pp. 3893-3905, 2012, doi: 10.1007/s10916-012-9861-z.

[12] G. Millar, A. M. Saks, and G. Tomlinson, “The practice of," J. Am. Med. Informatics Assoc., vol. 7, no. 1, pp. 1-20, 2000, doi: 10.1197/jamia.M1097.Affiliations.

[13] W. Newbrander, P. Ickx, F. Feroz, and H. Stanekzai, "Afghanistan's basic package of health services: its development and effects on rebuilding the health system.," Glob. Public Health, vol. 9 Suppl 1, no. July 2016, pp. S6-28, 2014, doi: 10.1080/17441692.2014.916735.

[14] J. W. Dearing and J. G. Cox, "Diffusion of innovations theory, principles, and practice," Health Aff., vol. 37, no. 2, pp. 183-190, 2018, doi: 10.1377/hlthaff.2017.1104.

[15] X. Zhang, P. Yu, J. Yan, and I. T. A. M. Spil, "Using diffusion of innovation theory to understand the factors impacting patient acceptance and use of consumer e-health innovations: a case study in a primary care clinic," pp. 1-15, 2015, doi: 10.1186/s12913-015-0726-2. 